\title{
LIV. On the production of light by heat
}

\section{John William Draper M.D.}

To cite this article: John William Draper M.D. (1847) LIV. On the production of light by heat , Philosophical Magazine Series 3, 30:202, 345-360, DOI: 10.1080/14786444708647190

To link to this article: http://dx.doi.org/10.1080/14786444708647190

册 Published online: 30 Apr 2009.

Submit your article to this journal 저

山 Article views: 13

Q View related articles $\square$

Citing articles: 1 View citing articles $\square$ 
LIV. On the Production of Light by Heat. By Joнn William Draper, M.D., Professor of Chemistry in the University of Nero York *.

A LTHOUGH the phænomenon of the production of light $A$ by all solid bodies, when their temperature is raised to a certain degree, is one of the most familiar in chemistry, no person so far as I know has hitherto attempted a critical investigation of it. The difficulties environing the inquiry are so great, that even among the most eminent philosophers a diversity of opinion has prevailed respecting some of the leading facts. Thus Sir Isaac Newton fixed the temperature at which bodies become self-luminous at $635^{\circ}$, Sir Humphry Davy at $812^{\circ}, \mathrm{Mr}$. Wedgwood at $94.7^{\circ}$, and $\mathrm{Mr}$. Daniell at $980^{\circ}$. As respects the nature of the light emitted there are similar contradictions. In some philosophical works of considerable repute, it is stated that when a solid begins to shine it first emits red and then white rays ; in others it is asserted that a mixture of blue and red light is the first that appears.

I have succeeded in escaping or overcoming many of the difficulties of this problem, and have arrived at satisfactory solutions of the main points; and as the experiments now to be described lead to some striking and perhaps unexpected analogies between light and heat, they commend themselves to our attention as having a bearing on the question of the identity of those imponderable principles. It is known that heretofore I have been led to believe in the existence of cardinal distinctions, not only between these but also other imponderable agents; and I may therefore state, that when this investigation was first undertaken, it was in the expectation that it would lead to results very different from those which have actually arisen.

The following are the points on which I propose to treat:-

1. To determine the point of incandescence of platinum, and to prove that different bodies become red-hot at the same temperature.

2. To determine the colour of the rays emitted by selfluminous bodies at different temperatures. This is done by the only reliable method-analysis by the prism.

From these experiments it will appear, that as the temperature rises the light increases in refrangibility; and making a due allowance for the physiological imperfection of the eye, the true order of the colours is red, orange, yellow, green, blue, indigo, violet.

* Communicated by the Author. 


\subsection{Prof. Draper on the production of Light by Heat.}

3. To determine the relation between the brilliancy of the light emitted by a shining body and its temperature.

Here we shall find that the intensity of the light increases far more rapidly than the temperature. For example, platinum at $2600^{\circ}$ emits almost forty times as much light as it does at $1900^{\circ}$.

As I prefer to give a complete description of the apparatus employed in these investigations after the general results are stated, it is sufficient here to understand that the source of light is in all instances a very thin strip of platinum 1.35 inch long and $\frac{1}{20}$ th of an inch wide, brought to the temperature under investigation by a voltaic current. Platinum was selected from its indisposition to oxidize, and its power of resisting a high temperature without fusion.

The slip of platinum, thus to be brought to different temperatures by an electric current of the proper force, was fastened at one end to an inflexible support, and at the other was connected with a delicate lever-index, which enabled me to determine its expansion, and thereby its temperature. For this purpose I have used the coefficient of dilatation of Dulong and Petit. The temperatures here given are upon the hypothesis of the invariability of that coefficient at all thermometric degrees; they are therefore to some extent in error.

By the aid of resisting wires of different lengths and a rheostat, I was able to vary the force of the electric current in the platinum, and thereby vary its temperature. My first attempts were to discover the point at which the metal begins to emit light.

The platinum and the voltaic battery were placed in a dark room, the temperature of which was $60^{\circ}$; and after I had remained there a sufficient length of time to enable my eyes to become sensible to feeble impressions of light, I caused the current to pass, gradually increasing its force, until the platinum was visible. In several repetitions of this experiment it was uniformly found that the index to which the platinum was attached stood at the eighth division when this took place. The metal had therefore dilated $\frac{1}{2} \frac{1}{2}$ of its length; the elevation of its temperature was about $917^{\circ}$, which added to the existing height of the thermometer, $60^{\circ}$, gives for the temperature of incandescence $977^{\circ} \mathrm{F}$.

To the correctness of the number it may be objected, that owing to the narrowness of the metallic strip it is not well calculated to make an impression on the eye when the light it emits is so feeble; nor can we take the dilatations given by the index, as representing the uniform temperature of the whole platinum, which must necessarily be colder near its 
points of support, by reason of the conducting power of the metals to which it is attached.

Physiological considerations would also lead us to suspect that the self-luninous temperature must vary with different eyes. The experiments of Bouguer, hereafter to be referred to, indisputably show that some persons are much more sensitive to the impressions of light than others. So far as my limited investigation of this matter has gone, I have not however found appreciable differences in the estimate of the temperature of incandescence. Different individuals, observing the platinum, have uniformly perceived it at the same time.

Against the number $977^{\circ}$ it may also be objected, that antimony melts at a much lower temperature, and yet emits light before it fuses. If this statement were true, it would lead us to believe that all bodies have not the same point of incandescence. But I think the experiments of Mr. Wedgwood on gold and earthenware are decisive of that question; and, moreover, I have reason to believe that the meltingpoint of antimony is much higher than commonly supposed.

With a view of determining directly whether different bodies vary in their point of incandescence, I took a clean gun-barrel, and having closed the touch-hole, exposed the following substances in it to the action of the fire:-platinum, chalk, marble, fluor spar, brass, antimony, gas-carbon, lead; each specimen was small; the platinum was in the form of a coil of stout wire.

When one of these bodies was placed in the gun-barrel and the temperature raised, it is clear that any difference in their point of incandescence would be detected by the eye. Thus, if the ignition of platinum required a higher degree than iron, on looking down the barrel the coil of wire should be dark, when the barrel itself begins to shine; or, if the platinum was incandescent first, the wire should be seen before the barrel is visibly hot; and these results might be corroborated by observing the inverse phænomena, when the barrel is taken from the fire and suffered to cool.

With respect to platinum, brass, antimony, gas-carbon and lead, they all became incandescent at the same time as the iron barrel itself. I could not discover the slightest difference between them, either in heating or cooling; and it is worthy of remark, that the lead was of course in the liquid condition. But the chalk and marble were visible before the barrel was red-hot, emitting a faint white light; and the fluor spar still more strikingly so, its light being of a beautiful blue; and even when the barrel had become bright red I could still see the spar, which had decrepitated to a coarse powder, by its 


\section{Prof. Draper on the production of Light by Heat.}

faint blue rays. In these cases it was not, however, incandescence but phosphorescence that was taking place. I infer then that all solids, and probably melted metals, shine at the same thermometric point.

The temperature of incandescence seems to be a natural fixed point for the thermometer; and it is very interesting to remark how nearly this point coincides with $1000^{\circ}$ of the Fahrenheit thermometer, when Laplace's coefficient for the dilatation of platinum is used. Upon that coefficient the point of incandescence is $1006^{\circ} \mathrm{F}$.

In view of these considerations, and recollecting that the number given by Daniell is $980^{\circ}$, and that of Wedgwood $947^{\circ}$, I believe that $977^{\circ}$ is not very far from the true temperature at which solids begin to shine. It is to be understood, of course, that this is in a very dark room.

I pass now to the second proposition. The rays emitted by the incandescent platinum were next received on a flint glass prism, placed so as to give the minimum deviation, and after dispersion viewed in a small telescope. A movement could be given to the telescope, which was read off on an annexed scale. However, instead of bringing the parts of the spectrum under measurement to coincide with the wires stretched across the field of the instrument, $I$ found it more satisfactory to determine them by bringing them to one or other of the edges of the field; a process well adapted to ascertain the position of the extreme rays, the faint light of which contrasted well with the darkness by which it was surrounded. They could not have been so accurately seen while the rest of the spectrum was in view; and as it was absolutely necessary to have fixed points of reference, that all the observations might be brought to a common standard of comparison, and as there are no fixed lines in the light of incandescence, such as are in the sunshine and daylight, I therefore previously determined the position of the fixed lines in a spectrum formed by a ray of reflected daylight which passed through a fissure $\frac{1}{g}$ th of an inch wide and one inch long, occupying exactly the position subsequently to be occupied by the incandescent platinum. Fig. 1 represents the results of this observation.

The strip of platinum was now placed in the position of the fissure which had given the spectrum fig. 1 , and its temperature was raised by the passage of a voltaic current. Although I could distinctly see the metal when the heat had reached about $1000^{\circ}$ by the naked eye, yet the loss of light in passing the prism and telescope was so great that $I$ found it necessary to carry the temperature to $1210^{\circ}$ before a satisfactory obserration could be made. At this point the spectrum extended 
Prof. Draper on the produciion of Light by Heat. $\quad 349$

from the position of the fixed line $B$ in the red, almost as far as the line $F$ in the green; the colours present being red,

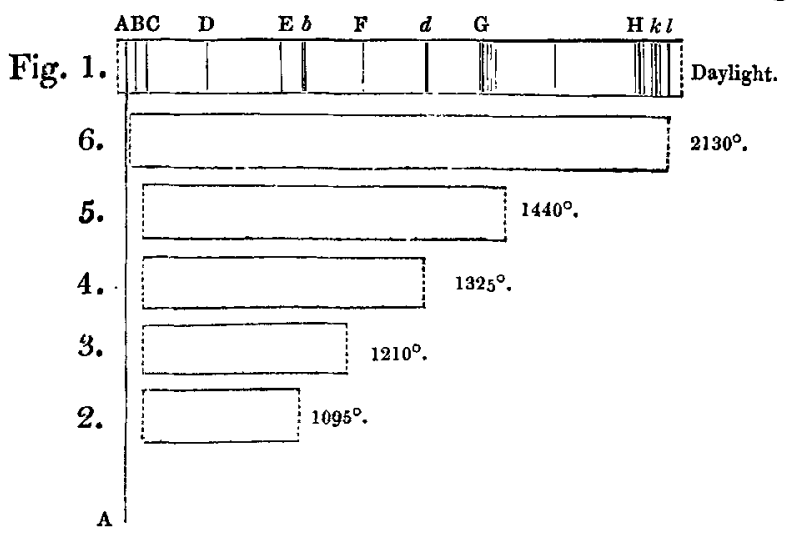

Spectra of incandescent platinum at different temperatures.

orange, and a tint which may be designated as gray. There was nothing answering to a yellow. The first rays visible through this apparatus may therefore be designated as red and greenish gray; the former commencing at the line B, and the latter continuing to $\mathrm{F}$. The magnitude and other relations of this spectrum are given in fig. 3 .

The voltaic current was now increased, and the temperature rose to $1325^{\circ}$. The red end of the spectrum remained nearly as before, but the more refrangible extremity reached to the position of the little fixed line $d$. Traces of the yellow were now visible; and, with a certain degree of distinctness, I could see red, orange, yellow, green, and a fringe of blue. Fig. 4 shows the result.

The temperature was now carried to $1440^{\circ}$. I thought the red extremity was advancing more to the line $A$ : the blue had undergone a well-marked increase. It reached considerably beyond the line $\mathrm{G}$, as shown in fig. 5 .

On bringing the platinum to $2130^{\circ}$ all the colours were present, and exhibited considerable brilliancy. 'Their extent was somewhat shorter than that of the daylight spectrum, as is seen in fig. 6.

Having thus by repeated experiments ascertained the continued extension of the more refrangible end as the temperature rose, it became necessary to obtain observations for points below $1210^{\circ}$, the limit of visibility through the telescope. I therefore carried the prism nearer to the platinum, and looking with the unassisted eye directly through it at the refracted image, I found it could be distinctly seen at a temperature as 


\section{Prof. Draper on the production of Light by Heat.}

low as $1095^{\circ}$. Under these circumstances the total length could not be compared by direct measurenient with the other observations, and the result given in fig. 2 is from the best judgement $I$ was able to form: the colours were red and greenish-gray.

The gray rays emitted by platinum just beginning to shine appear to be more intense than the red; at all events the wires in the field of the telescope are more distinctly seen upon them than upon the other colour. I give them the designation of gray, for they appear to approach that tint more closely than any other ; and yet it is to be remarked that they are occupying the position of the yellow and green regions.

Already we have encountered a fact of considerable importance. The idea, that as the temperature of a body rises it begins to emit rays of increasing refrangibility, has obviously to be taken with a certain restriction. Instead of first the red, then the orange, then the yellow, \&c. rays, in succession making their appearance, in which case the spectrum should regularly increase in length as the temperature rises, we here find, at the very first moment it is visible to the eye, it yields a spectrum reaching from the fixed line $\mathrm{B}$ to nearly $\mathrm{F}$; that is to say, equal to about two-thirds the whole length of the interference spectrum, and almost one-half of the prismatic.

It is to be remarked, that while the more refrangible end undergoes a great expansion, the otber extremity exhibits a corresponding though a less change. As very important theoretical conclusions depend on the proper interpretation of this fact, we must not forget that, to a certain extent, it may be an optical deception, arising from the increased brilliancy of the light. While the rays are yet feeble, the extreme terminations may be so faint that the eye cannot detect them; but as the intensity rises, they becone better marked, and an apparent elongation of the spectrum is the consequence.

It is agreed by optical writers, that to the human eye the yellow is the brightest of the rays. In the prismatic spectrum the true relationship of the colours is not perceived, because the less refrangible are crowded together, and the more refrangible unduly spread out. But in the interference spectrum, where the colours are arranged side by side in the order of their wave-lengths, the centre is occupied by the most luminous portion of the yellow; and from this point the light declines away on one side in the reds, and on the other in the blues, the terminations being equidistant from the centre of the yellow space.

Now if the rays coming from shining platinum were passed through a piece of glass, on which parallel lines had been 
drawn with a diamond point, so as to give an interference spectrum, even admitting the general results of the foregoing experiments to be true, viz. that as the temperature rises rays of a higher refrangibility are emitted, it is obvious that it by no means follows that the first ray visible should be the extreme red. Our power of seeing that depends on its having a certain intensity. Even when it has assumed that extreme brilliancy which it has in a solar beam it is barely visible. We ought therefore to expect that rays of a higher refrangibility should first be seen, because they act more energetically on our organ of vision; and as the temperature rises, the spectrum should undergo a partial elongation in the direction of its red extremity.

I may here remark, that the general result of these experiments coincides exactly with that of $M$. Melloni respecting heat at lower thermometric points. In his second memoir*, he shows that when the rays from copper at $390^{\circ}$ and from incandescent platinum are compared by transmission through a rock-salt prism, as the temperature rises the refrangibility of the calorific emanations correspondingly increases. 'Those philosophers who regard light and heat as the same agent, will therefore see in this coincidence another argument in favour of their opinion.

In view of the foregoing facts I conclude, that, as the temperature of an incandescent body rises, it emits rays of light of an increasing refrangibility; and that the apparent departure from this law, discovered by an accurate prismatic analysis, is due to the special action of the eye in performing the function of vision.

As the luminous effects are undoubtedly owing to a vibratory movement executed by the molecules of the platinum, it seems from the foregoing considerations to follow, that the frequency of those vibrations increases with the temperature.

In this observation I am led by the principle, that " to a particular colour there ever belongs a particular wave-length, and to a particular wave-length there ever belongs a particular colour;" but in the analysis of the spectrum made by Sir $D$. Brewster by the aid of absorptive media, this principle is indirectly controverted; that eminent philosopher showing that red, yellow, blue, and consequently white light, exist in every part of the spectrum. This must necessarily take place when a prism which has a refracting face of considerable magnitude is used; for it is obvious that a ray falling near the edge, and one falling near the back, after dispersion, will paint their several spectra on the screen; the colours of the one not co-

* Taylor's Scientific Memoirs, vol. i. p. 56. 


\section{Prof. Draper on the production of Light by Heat.}

inciding with, but overlapping the colours of the other. In such a spectrum there must undoubtedly be a general commixture of the rays; but may we not fairly inquire whether, if an elementary prism were used, the same facts would hold good; or, if the anterior face of the prism were covered by a screen, so as to expose a narrow fissure parallel to the axis of the instrument, would there be found in the spectrum it gave every colour in every part, as in Sir David Brewster's original experiment?" M. Melloni has shown how this very consideration complicates the phænomena of radiant heat; and it would seem a very plausible suggestion that the effect here pointed out must occur in an analogous manner for the phænomena of light.

I proceed now to the third branch of the inquiry,-to examine the relation between the temperatures of self-luminous bodies and the intensity of the light they emit, premising it with the following considerations.

The close analogy which is traced between the phænomena of light and radiant heat lends countenance to the supposition, that the law which regulates the escape of caloric from a body will also determine its rate of emission of light. Sir Isaac Newton supposed that whilst the temperature of a body rose in arithmetical progression, the amount of heat escaping from it increased in a geometrical progression. The fallacy of this was subsequently shown by Martin, Erxleben, and Delaroche; and finally Dulong and Petit gave the true law, "when a body cools in vacuo, surrounded by a medium whose temperature is constant, the velocity of cooling for excess of temperature in arithmetical progression increases as the terms of a geometrical progression, diminished by a constant quantity." The introduction of this constant depends on the operation of the theory of exchanges of heat; for a body, when cooling under the circumstances here given, is simultaneously receiving back a constant amount of heat from the medium of constant temperature.

Whilst Newton's law represents the rate of cooling of bodies, and therefore the quantities of heat they enit, when the range of temperature is limited, and the law of Dulong and Petit holds to a wider extent, there are in our inquiry certain circumstances to be taken into account not contemplated by those philosophers. Dulong and Petit throughout their memoir regard radiant heat as a homogeneous agent, and look upon the theory of exchanges, which is indeed their startingpoint and guide, as a very simple affair. But the progress of this department of knowledge since their times has shown, that precisely the same modifications as are found in the co- 
Prof. Draper on the production of Light by Heat. 353

lours of light, occur also for heat; a fact conveniently designated by the phrase "ideal coloration of heat;" and further, that the colour of the heat emitted depends upon the temperature of the radiating source. It is one thing to investigate the phænomena of the exchanges of heat-rays of the same colour, and another when the colours are different. A perfect theory of the exchanges of heat must include the principle of ideal coloration, and, of course, so too must a law of cooling applicable to any temperature.

There is another fact to some extent considered by Dulong and Petit, but not of such weight in their investigations, where the range of temperature was small, as in ours, where it rises as high as nearly $3000^{\circ} \mathrm{F}$.; I mean the difference of specific heat of the same body at different temperatures. At the high temperatures considered in this memoir, there cannot be a doubt that the capacity of platinum for heat is far greater than that at a low point. This therefore must control its rate of calorific emission, and probably that for light also.

From these and similar considerations, we should be prepared to discover that as the temperature of an incandescent solid rises, the intensity of the light emitted increases very rapidly.

I pass now to the experimental proofs which substantiate the foregoing reasoning.

The apparatus employed as the source of the light and measure of the temperature was the same as in the preceding experiments, - a strip of platinum, brought to a known temperature by the passage of a voltaic current of the proper force, and connected with an index which measured its expansion.

The principle upon which $I$ have determined the intensities of the light is that first described by Bouguer, and recently intraduced by M. Masson. After many experiments I have been led to conclude that this is the most accurate method known.

Any gne who will endeavour to determine the intensities of lights by Rumford's method of contrasting shadows, or by that of equally illuminated surfaces, will find, when every precaution has been used, that the results of repeated experiments do not accord. There is moreover the great defect, that where the lights differ in colour it is impossible to obtain reliable measures, except by resorting to such contrivances as that described by me*.

Bouguer's principle is far more exact; and where the lights differ in colour, that difference actually tends to make the

* Phil. Mag., August 1844.

Phil. Mag. S. 3. Vol. 30. No. 202. May 184.7. 2 B 


\section{Prof. Draper on the production of Light by Heat.}

result more perfect. As it is not generally known, I will indicate the nature of it briefly.

Let there be placed at a certain distance from a screen of white paper, a candle so arranged as to throw the shadow of a ruler, or other opake body, on the screen. If a second candle be placed also in front of the paper and nearer than the former, there is a certain distance at which its light completely obliterates all traces of the shadow. This distance is readily found ; for the disappearance of the shadow can be determined with considerable exactness. When the lights are equal, Bouguer found that the relative distances were as $1: 8$; he inferred therefore, correctly, that in the case of his eye, the effect of a given light was impercentible when it was in presence of another sixty-four times as intense. The precise number differs according to the sensibility of different eyes, but for the same organ it is constant.

Upon a paper screen I threw the shadow of a piece of copper, which intercepted the rays of the incandescent platinum : then taking an Argand lamp, surrounded by a cylindrical metal shade through an aperture in which the light passed, and the flame of which I had found by previous trial would continue for an hour almost of the same intensity, I approached it to the paper until the shadow cast by the copper disappeared. The distance at which this took place was then measured, and the temperature of the platinum determined.

The temperature of the platinum was now raised; the shadow became more intense, and it was necessary to bring the Argand lamp nearer before it was effaced. When this took place the distance of the lamp was again measured, and the temperature of the platinum again determined.

In this manner I obtained several series of results, one of which is given in the following table. 'They exhibited a more perfect accordance among each other than I had anticipated.

Table of the Intensity of Light emitted by Platinum at different Temperatures.

\begin{tabular}{|c|c|c|c|c|}
\hline \multirow{2}{*}{$\begin{array}{l}\text { Temperature of } \\
\text { the platinum. }\end{array}$} & \multicolumn{2}{|c|}{ Distance of Argand lamp. } & \multirow{2}{*}{ Mean. } & \multirow{2}{*}{$\begin{array}{l}\text { Intensity of } \\
\text { light. }\end{array}$} \\
\hline & Experinient 1. & Experiment 2. & & \\
\hline 980 & & & & 0.00 \\
\hline 1900 & $54 \cdot 00$ & $54 \cdot 00$ & $34 \cdot 00$ & 0.34 \\
\hline 2015 & $39 \cdot 00$ & $41 \cdot 00$ & 40.00 & 0.62 \\
\hline 2130 & $24 \cdot 00$ & $24 \cdot 00$ & $24 \cdot 00$ & $1 \cdot 73$ \\
\hline 2245 & $18 \cdot 00$ & $19 \cdot 00$ & $18 \cdot 50$ & 2.92 \\
\hline 2360 & $14 \cdot 50$ & $15 \cdot 50$ & $15 \cdot 00$ & $4 \cdot 40$ \\
\hline 2475 & $11 \cdot 50$ & 12.00 & 11.75 & $7 \cdot 24$ \\
\hline 2590 & 9.00 & $9 \cdot 00$ & $9 \cdot 00$ & $12 \cdot 34$ \\
\hline
\end{tabular}


Prof. Draper on the production of Light by Heat. 355

The intensity of the light of the platinum is of course inversely proportional to the square of the distance of the Argrand lamp at the moment of the obliteration of the shadow.

In this table the first column gives the temperatures under examination in Fahrenheit degrees; the second and third the distances of the Argand lamp from the screen, in English inches, in two different sets of experiments; the fourth the mean of the two; and the fifth the corresponding intensity of the light.

From this it is at once perceived, that the increases in the intensity of the light, though slow at first, become very rapid as the temperature rises. At $2590^{\circ}$ the brilliancy is more than thirty-six times as great as it is at $1900^{\circ}$.

Thus, therefore, the theoretical anticipation which we founded on the analogy of light and heat is completely verified ; and we discover that as the temperature of a self-luminous solid rises, it emits light in a greater proportion than would correspond to the mere difference of temperature. 'To place that analogy in a still more striking point of view, I will here introduce some experiments $I$ have made in relation to radiant heat. No chemist, so far as I am aware, has hitherto published results for high temperatures, or endeavoured to establish, through an extensive scale, the principle of Delaroche, that "the quantity of heat which a hot body gives off in a given time by way of radiation to a cold body, situated at a distance, increases, other things being equal, in a progression more rapid than the excess of the temperature of the first above that of the second."

As my object on the present occasion is chiefly to illustrate the remarkable analogy between light and heat, the experiments now to be related were arranged so as to resemble the foregoing; that is to say, as in determining the intensities of light emitted by a shining body at different temperatures, I had received the rays upon a screen placed at an invariable distance, and then determined their value by photometric methods; so, in this case, I received the rays of heat upon a screen placed at an invariable distance, and determined their intensity by thermometric methods. In this instance the screen employed was in fact the blackened surface of the thermoelectric pile. It was placed at a distance of about one inch from the slip of incandescent platinum, a distance sufficient to keep it from any disturbance from the stream of hot air arising from the metal ; care also was taken that the multiplier itself was placed so far from the rest of the apparatus, that its astatic needles could not be affected by the voltaic current 2 B 2 


\section{Prof. Draper on the production of Light by Heat.}

igniting the platinum, or the electro-magnetic action of the wires used to modify the degrees of heat.

The experiments were conducted as follows:-The needles of the thermo-multiplier standing at the zero of their scale, the voltaic current was passed through the platinum, which immediately rose to the corresponding temperature, and radiated its heat to the face of the pile. The instant the current passed, the needles of the multiplier moved, and kept steadily advancing upon the scale. At the close of one minute, the deviation of the needle and the temperature of the platinum were simultaneously noted, and then the voltaic current was stopped.

Sufficient time was now given for the needle of the multiplier to come back to zero. This time varied in the different cases, according to the intensity of the heat to which the pile had been exposed: in no instance, however, did it exceed six minutes, and in most cases was much less. A little consideration will show that the usual artifice employed to drive the needles back to zero, by warming the opposite face of the pile, was not admissible in these experiments.

The needles having regained their zero, the platinum was brought again to a given temperature, and the experiment conducted as before. The following table exhibits a series of these results.

Table of the Intensity of Radiant Heat emitted by Platinum at different Temperatures.

\begin{tabular}{|c|c|c|c|}
\hline $\begin{array}{c}\text { Temperature of } \\
\text { the platinum. }\end{array}$ & \multicolumn{2}{|c|}{ Intensity of heat emitted. } & Mean. \\
\hline & Experiment 1. & Experiment 2. & \\
\hline 980 & & & \\
1095 & 75 & $1 \cdot 00$ & $\cdot 87$ \\
1210 & $1 \cdot 00$ & $1 \cdot 20$ & $1 \cdot 10$ \\
1325 & $1 \cdot 40$ & $1 \cdot 60$ & $1 \cdot 50$ \\
1440 & $1 \cdot 60$ & $2 \cdot 00$ & $1 \cdot 80$ \\
1555 & $2 \cdot 20$ & $2 \cdot 20$ & $2 \cdot 20$ \\
1670 & $2 \cdot 75$ & $2 \cdot 85$ & $2 \cdot 80$ \\
1785 & $3 \cdot 65$ & $3 \cdot 75$ & $3 \cdot 70$ \\
1900 & $5 \cdot 00$ & $50 \cdot 0$ & $5 \cdot 00$ \\
2015 & $6 \cdot 70$ & $6 \cdot 90$ & $6 \cdot 80$ \\
2130 & $8: 60$ & $8 \cdot 60$ & $8 \cdot 60$ \\
2245 & $10 \cdot 00$ & $10 \cdot 00$ & $10 \cdot 00$ \\
2360 & 12.50 & $12 \cdot 50$ & $12 \cdot 50$ \\
& 15.50 & 15.50 & 15.50 \\
\hline
\end{tabular}

In this table the first column gives the temperatures of the 
platinum in Fahrenheit degrees; the second and third two sets of experiments, expressing the arc passed over by the needle at the close of a radiation lasting for one minute, each number being the mean of several successive trials; and the fourth the mean of the two. It therefore gives the radiant effect of the incandescent platinum upon the thermo-multiplier for the different temperatures.

Of course it is understood that I here take the angular deviations of the needle as expressing the force of the thermoelectric current, or in other words, as being proportional to the temperatures. This hypothesis, it is known, is admissible.

It therefore appears that the quantity of heat radiated by incandescent platinum at $980^{\circ}$ being taken as unity, it will have increased at $1440^{\circ}$ to 2.5 ; at $1900^{\circ}$ to 7.8 ; and at $2360^{\circ}$ to $17 \cdot 8$, nearly: the rate of increase is therefore very rapid. Further, it may be renarked, as illustrative of the same fact, that the increased quantity of heat radiated by a mass of platinum in passing from $1000^{\circ}$ to $1300^{\circ}$, is nearly equal to the amount it gives out in passing from common temperatures up to $1000^{\circ}$.

I cannot here express myself with too much emphasis on the remarkable analogy between light and heat which these experiments reveal. The march of the phænomena in all their leading points is the same in both cases. The rapid increase of effect as the temperature rises is common to both.

It is not to be forgotten, however, that in the case of light we necessarily measure its effects by an apparatus which possesses special peculiarities. The eye is insensible to rays which are not comprehended within certain limits of refrangibility. In these experiments, it is requisite to raise the temperature of the platinum almost to $1000^{\circ}$ before we can discover the first traces of light. Measures obtained under such circumstances are dependent on the physiological action of the visual organ itself, and hence their analogy with those obtained by the thermometer becomes more striking, because we should scarcely have anticipated that it could be so complete.

\section{Description of the apparatus employed in the foregoing experiments.}

The source of light is in all instances a slip of platinum foil 1.35 inch long, and $\frac{1}{2} 0$ th of an inch broad, ignited by the passage of a voltaic current, and placed in such a position that its dilatation could be measured by the movements of an index over a graduated scale. 


\section{Prof. Draper on the production of Light by Heat.}

In fig. 7, $a b$ represents the slip of platinum, the upper end of which is soldered to a stout and short copper pin $a$, firmly sunk in a block of wood $c$, which is immoveably fastened on the basis $d d$ of the instrument. A cavity $e$, half an inch in diameter, is sunk in the block $c$, and into this cavity the pin $a$ projects; so that when the cavity is filled with mercury, a voltaic current may be passed through the pin and down the platinum.

Fig. 7.

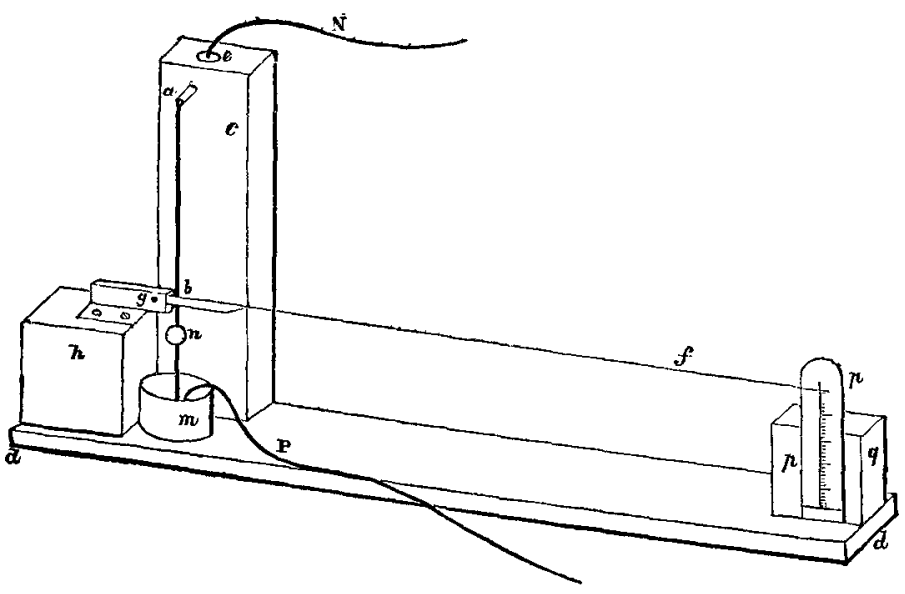

The other extremity of the platinum $b$ is fastened to a delicate lever $b f$, which plays on an axis at $g$, the axis working in brass holes supported on a block $h$. Immediately beneath the platinum strip, and in metallic communication with it, a straight copper wire dips down into the mercury cup $m$; on this wire there is a metal ball $n$, weighing about 100 grains. The further end of the index plays over a graduated ivory scale $p p$, which is supported on a block $q$, and can be moved a little up and down, so as to bring its zero to coincide with the index at common temperatures.

The action of the instrument is readily understood. In the mercury cup $e$ dip one of the wires $N$ of a Grove's battery of three or four pairs, the other wire $P$ being dipped into the cup $m$. 'The current passes through the platinum, which immediately expands, the weight $n$ lightly stretching it. The index $f$ moves promptly over the scale, indicating the amount of expansion, and therefore the degree of heat. Remove the wire $\mathrm{N}$ out of its mercury cup $e$, the platinum instantly becomes cold, and pulls the lever to the zero point.

When the platinum is thin, so as to be quite flexible at the 
point $b$, where it is fastened to the index, the movements take place with such promptitude and precision as to leave rothing to be desired. When the heat has been very high and long continued, the limit of elasticity of the platinum is somewhat overpassed, and it suffers a slight permanent extension. But as the ivory scale $p p$ can slide up and down a little, the index is readily re-adjusted to the zero point.

The temperature of the platinum depends entirely on the force of the current passed through it. By intervening coils of brass wire of lengths adjusted beforehand, so as to resist the current to a given extent, any desired temperature may be reached. I found it convenient to intervene in the course of the current one of Prof. Wheatstone's rheostats, so as to be able to bring the index with precision to any degree, notwithstanding slight changes in the force of the voltaic battery.

The following are the dimensions and measures of the instrument $I$ have used:-Length of the platinum strip, 1.35 inch; length of the part actually ignited, $1 \cdot 14$ inch; width of ditto, ${ }^{3}$ th of an inch; length of the index from its centre of motion to the scale, $7 \cdot 19$ inches; distance of the centre of motion of index from the insertion of the platinum at the point $b, .22$ inch; multiplying effect of the index, 32.68 times; length of each division on the ivory scale, 021 inch. From this it would appear, by a simple calculation, using the coefficient of dilatation of platinum given by Dulong and Petit, that each of the divisions here used is equal to 114.5 Fahrenheit degrees. For the sake of perspicuity I have generally taken them at $115^{\circ}$.

The Grove's battery I have employed has platinum plates three inches long and three quarters wide; the zinc cylinders are two inches and a half in diameter, three high, and onethird thick. As used in these experiments, it could maintain a current nearly uniform for an hour. I commonly employed four pairs.

Among writers on optics, it has been a desideratum to obtain an artificial light of standard brilliancy. The preceding experiments furnish an easy means of supplying that want, and give us what might be termed a "unit-lamp." A surface of platinum of standard dimensions, raised to a standard temperature by a voltaic current, will always emit a constant light. A strip of that metal, one inch long and $\frac{1}{2} \frac{1}{0}$ th of an inch wide, connected with a lever by which its expansion 
360 On the Acid contained in the North Americun Cotumbite.

might be measured, would yield at $2000^{\circ}$ a light suitable for most purposes. Moreover, it would be very easy to form from it an available photometer, by screening portions of the shining surface. An ingenious artist would have very little difficulty, by taking advantage of the movements of the lever, in making a self-acting apparatus, in which the platinum should be maintained at a uniform temperature, notwithstanding any change taking place in the voltaic current.

University, New York, Feb. 27, 1847.

LV. On the Acid contained in the North American Columbite. By Henry Rose*.

THE columbite of North America has the same crystalline form as that from Bodenmais in Bavaria, but is distinguished from it in general by a far lower specific gravity; however, we find the same difference in the specific gravity of the American mineral as occurs in the different crystals of the Bodenmais columbite. The lightest crystals from the last locality have the same specific gravity $(5 \cdot 704)$ as the heaviest crystals from North America (5:708).

I have already communicated two analyses of North American columbites, of one of which however it was doubtful whether it came from America. The following analysis of American columbite was made by $M$. Grewink in my laboratory; it yielded,-

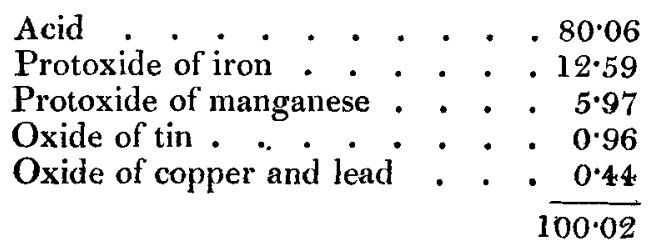

The specific gravity in fragments was $5 \cdot 323$; in powder, $5 \cdot 3202$.

This columbite comes netrest in composition and also in specific gravity to that examined by M. Schlieper.

I have on a former occasion shown that the different specific gravity of the crystals of the Bavarian columbite was owing to the different proportions of niobic and pelopic acids which are found in the different crystals. The specific gravity of these two acids is widely different, but unequally so, according to the temperatures to which they have been exposed previous to weighing.

Owing to want of material, I found it impossible to make a

$$
\text { * Translated from Poggendorf's Annalen. }
$$

